\title{
A case report of nodal marginal zone lymphoma: Diagnosis and management
}

\begin{abstract}
Nodal marginal zone B-cell lymphoma (NMZL) is a rare subtype of non-Hodgkin lymphoma, usually presented at advanced stage. The treatment for NMZL is currently consistent with follicular lymphoma which includes observation for asymptomatic patients to cyclophosphamide, doxorubicin, vincristine, prednisone (CHOP) or CHOP plus rituximab regimen, fludarabine or bendamustine and rituximab (B-R) for advanced, symptomatic patients. The B-R regimen is effective and could be evaluated further as the first-line therapy.
\end{abstract}

Keywords: Bendamustine, nodal marginal zone B-cell lymphoma, rituximab

\section{INTRODUCTION}

Marginal zone lymphomas are B-cell origin and account for about $5 \%-10 \%$ of lymphomas. As per the WHO classification, mucosa-associated lymphoid tissue (MALT) lymphoma, splenic marginal zone B-cell lymphoma (SMZL), and nodal marginal zone B-cell lymphoma (NMZL) are three main types of marginal zone lymphomas.

Extra-NMZL, also known as MALT lymphoma, is a low-grade indolent lymphoma diagnosed commonly in the sixth decade. The common site of involvement is stomach but it can also be developed in salivary gland, thyroid, and lung. Most MALT gastric lymphomas are linked to Helicobacter pylori infection and are treated with $\mathrm{H}$. pylori-eradicating treatment. $\mathrm{H}$. pylori negative MALT lymphomas are treated with radiotherapy for early localized disease and single or multiple-drug chemotherapy for advanced disease.

SMZL is a rare lymphoma mostly involved in spleen and bone marrow. The median age of diagnosis is 69 years. It is associated with autoimmune disease and hepatitis $C$ virus infection. This is indolent lymphoma, but $5 \%-10 \%$ cases can be transformed into high-grade B-cell lymphoma. Spleen histopathology is considered a gold standard for diagnosis;

\begin{tabular}{|l|c|}
\hline \multicolumn{2}{|c|}{ Access this article online } \\
\hline \multirow{2}{*}{$\begin{array}{l}\text { Website: } \\
\text { www.asjo.in }\end{array}$} & Quick Response Code \\
\hline \multirow{2}{*}{$\begin{array}{l}\text { DOI: } \\
\text { 10.4103/ASJO.ASJO_80_16 }\end{array}$} & \\
&
\end{tabular}

however, if splenectomy is not done, then bone marrow histology, clinical findings, and the immunophenotype can assist with diagnosis. ${ }^{[1]}$

NMZL is a slow-growing rare disease, represents $1 \%$ of all non-Hodgkin lymphoma (NHL), found mainly in older women with an average age of around 60 years. It usually stays in the lymph nodes although lymphoma cells can also involve bone marrow. Most patients present with stage III/IV stage. Cells express monoclonal surface immunoglobulin (IgM > IgG > IgA) as well as CD19, CD20, CD79a and are negative for CD10 and CD23. The evaluation and staging is similar to the follicular lymphoma which includes observation for asymptomatic patients to chemotherapy such as cyclophosphamide, doxorubicin, vincristine, prednisone (CHOP) or CHOP plus rituximab (R-CHOP) regimen or bendamustine and rituximab (B-R) regimen. The 5-year survival is $55 \%-79 \%$.

\section{Dilip Sandipan NIKam}

Department of Radiotherapy and Oncology, Cama and Albless Hospitals, Grant Government Medical College and Sir J. J.

Group of Hospitals, Mumbai, Maharashtra, India

Address for correspondence: Dr. Dilip Sandipan Nikam, Department of Radiotherapy and Oncology, Cama and Albless Hospitals, Grant Government Medical College and Sir J. J. Group of Hospitals, Mumbai, Maharashtra, India.

E-mail: docnik128@yahoo.com

This is an open access article distributed under the terms of the Creative Commons Attribution-NonCommercial-ShareAlike 3.0 License, which allows others to remix, tweak, and build upon the work non-commercially, as long as the author is credited and the new creations are licensed under the identical terms.

For reprints contact: reprints@medknow.com

How to cite this article: Nikam DS. A case report of nodal marginal zone lymphoma: Diagnosis and management. Asian J Oncol 2017;3:78-80. 


\section{CASE REPORT}

A 58-year-old postmenopausal female, with three living children, consulted for chief complaint of multiple swellings in bilateral neck, axilla, and inguinal region for 7 months and difficulty in mouth opening and swallowing, gradually progressive. In general, the examination was within normal limits, except multiple lymphadenopathy in bilateral neck, axilla, and inguinal region largest lesion measuring $15 \mathrm{~cm} \times 11 \mathrm{~cm}$ in left parotid region and left upper neck region. Per abdominal examination revealed no organomegaly; the patient's hematological and biochemical workup was within normal limits. Urine routine and microscopic examination was within normal limits. Two-dimensional echo was within normal limits. Positron emission tomography-computed tomography (PET-CT) revealed metabolically active extensive supra- and infra-diaphragmatic lymphadenopathy including bilateral cervical level II, III, IV, V, left supraclavicular, bilateral axillary, right lower pretracheal, prevascular, left internal mammary, subcarinal, gastrohepatic, paraaortic, precaval, retrocaval, aortocaval, bilateral internal iliac, external iliac, and inguinal. The largest lymph node mass was measuring $9.2 \mathrm{~cm} \times 12.2 \mathrm{~cm} \times 7.8 \mathrm{~cm}$ in the left parotid region [Figure 1]. No other metabolically active lesion suggesting extranodal involvement is seen.

The right cervical node excision biopsy done on October 2015 was reported as a low-grade B-cell NHL, in favor of NMZL. On immunohistochemistry, CD23 highlights the retained but distorted native follicular dendritic cell network throughout the lymph node; CD20 highlights the large expansile nodular and confluent area of B-lymphoid cells. Some of CD20 cells are positive for Bcl-6. Cyclin D1 and Bcl-2 are negative. MIB-1 labeling index for areas corresponding to $\mathrm{Bcl}-6$ and $\mathrm{CD} 23$ highlights very high labeling index representing distorted native germinal centers, while areas corresponding to Bcl-6 negative and CD20 positive show labeling index approximately $10 \%-15 \%$.

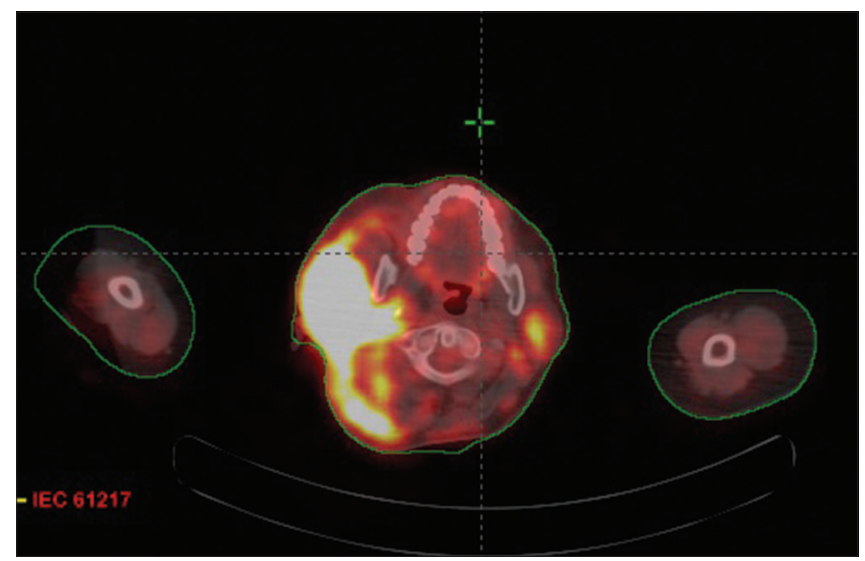

Figure 1: Prechemotherapy positron emission tomography-computed tomography image
Bone marrow examination revealed hypercellular bone marrow with $10 \%$ lymphocytes but not involved by lymphoma.

The patient was started on B-R regimen from October 27, 2015, with peg-Gcf support. The patient received three cycles of B-R regimen last dose on December 14, 2015. The postchemotherapy PET-CT report showed complete metabolic response [Figure 2].

\section{DISCUSSION}

NMZL, or also known as monocytoid B-cell lymphoma, is a slow-growing rare disease, represents $1 \%$ of all NHL. It is mainly found in older women with median age is 60 years. It usually involves lymph nodes although one-third of patients were found with bone marrow involvement. Around 70\% patients present with advanced III/IV stage. The lymph node effacement occurs partially or totally with monocytoid-like B-cells infiltration. The follicular meshwork dendritic network favors the diagnosis. The cells express monoclonal surface immunoglobulin (IgM > $\lg G>\operatorname{IgA})$ as well as CD19, CD20, CD22, CD79a, bcl2 and are negative for CD5, CD10, CD23, and $\mathrm{CD} 25$.

As per the National Comprehensive Cancer Network guidelines, the evaluation and treatment for NMZL is currently consistent with follicular lymphoma that includes observation for asymptomatic patients to chemotherapy such as CHOP or $\mathrm{R}$-CHOP regimen or B-R regimen. NMZL is only curable during its early stages and is more aggressive than SMZL. The transformation to diffuse large B-cell lymphoma can occur. The 5 -year survival is $55 \%-79 \% .{ }^{[2]}$

This patient is a 58-year-old female with stage III disease and presented with prominent neck node mass which is most

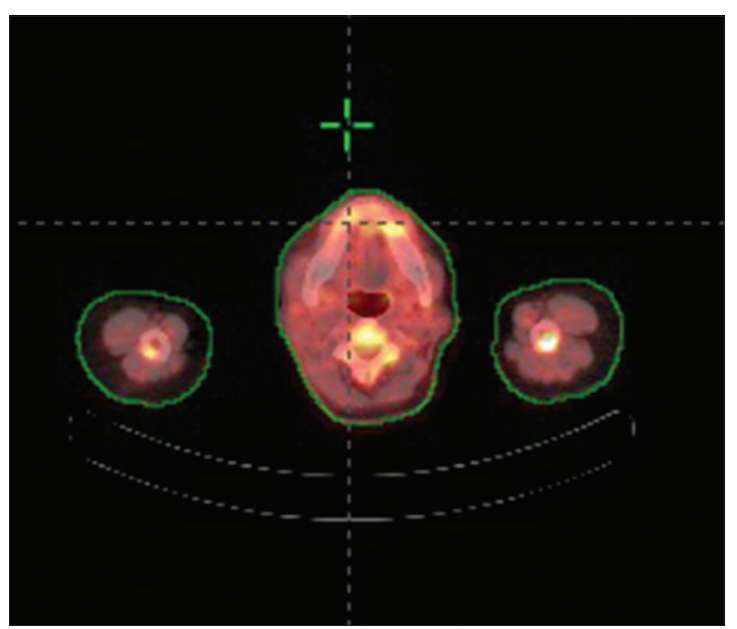

Figure 2: Postchemotherapy positron emission tomography-computed tomography image 
common site for involvement. Our patients' histopathological findings including morphological features and CD expression were similar to those of previously published literatures.

The chemotherapy studies have shown differing response rate to different chemotherapy combinations. The anthracycline-based CHOP regimen is most commonly used to treat NMZL but no convincing data available till date. Rummel et al. compared B-R with R-CHOP as the first-line treatment for patients with indolent and mantle cell lymphomas; they concluded that previously untreated patients with indolent lymphoma, B-R can be considered as a preferred first-line treatment approach to R-CHOP because of increased progression-free survival and fewer toxic effects. ${ }^{[3]}$

Retrospective analysis by Mondello et al. compared the efficacy and safety of both B-R regimen and R-CHOP regimen in clinical practice in low-grade-NHL patients. They concluded that $\mathrm{B}-\mathrm{R}$ regimen is an effective and less toxic therapeutic option than R-CHOP in clinical practice. ${ }^{[4]}$

This patient has received three cycles of B-R regimen with complete metabolic response. The chemotherapy-related side effects were manageable. The patient is on regular follow-up.

The prognostic factors for all MZL were advancing age and presence of B symptoms while male sex and stage of the disease were associated with NMZL and MALT subtypes.

The impact of clinical characteristics and survival outcomes for the subtypes of MZL was studied by Olszewski and Castillo, which revealed the prognosis was significantly better for patients with MALT subtype lymphoma (5-year relative survival rate of $88.7 \%$; $P<0.0001$ ) compared with those with the SMLZ or NMZL subtypes (5-year relative survival rates of $79.7 \%$ and $76.5 \%$, respectively). There was evidence of improved outcomes in patients with NMZL and MALT lymphomas $(P<0.0001)$, with no difference noted in patients with SMZL $(P=0.56) .{ }^{[5]}$

\section{CONCLUSION}

NMZL is a slow-growing rare disease where patients with early-stage asymptomatic disease are observed. The advanced symptomatic disease can be treated effectively with B-R regimen. The $B-R$ regimen is effective and could be evaluated further as the first-line therapy.

Financial support and sponsorship

Nil.

Conflicts of interest

There are no conflicts of interest.

\section{REFERENCES}

1. Arcaini L, Paulli M. Splenic marginal zone lymphoma: Hydra with many heads? Haematologica 2010;95:534-7.

2. Joshi M, Sheikh H, Abbi K, Long S, Sharma K, Tulchinsky M, et al. Marginal zone lymphoma: Old, new, targeted, and epigenetic therapies. Ther Adv Hematol 2012;3:275-90.

3. Rummel MJ, Niederle N, Maschmeyer G, Banat GA, von Grünhagen U, Losem C, et al. Bendamustine plus rituximab versus $\mathrm{CHOP}$ plus rituximab as first-line treatment for patients with indolent and mantle-cell lymphomas: An open-label, multicentre, randomised, phase 3 non-inferiority trial. Lancet 2013;381:1203-10.

4. Mondello P, Steiner N, Willenbacher W, Wasle I, Zaja F, Zambello R, et al. Bendamustine plus rituximab versus R-CHOP as first-line treatment for patients with indolent non-Hodgkin's lymphoma: Evidence from a multicenter, retrospective study. Ann Hematol 2016;95:1107-14.

5. Olszewski AJ, Castillo JJ. Survival of patients with marginal zone lymphoma: Analysis of the surveillance, epidemiology, and end results database. Cancer 2013;119:629-38. 